\title{
PNIRSAsia-Pacific
}

架起东西方之间的桥梁

心理神经免㡯学是研究神
经系统与免度系统之间䏍
系的学科, 它在应激、正
念、古法健身和饮食干预
等领域率先取得了重大发
现。心理神经免度学研究
协会(PNIRS) 2012 年成立
了中国分会, 并在其获得
成功之后, 扩展到了整个
亚洲和大洋洲地区・伊利
诺伊大学的Keith W. Kelley
及其合作者回顾了协会的
成长历程以及亚洲和大洋
洲各国家及地最近对生
物医学研究的贡献。

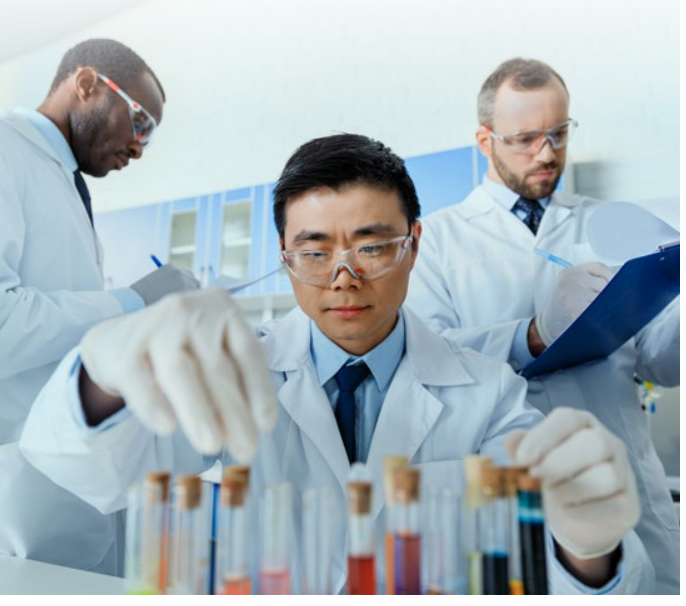

心理神经免疫学是生物医学研究 中相对较新的领域, 它探索神经 系统与免疫系统之间的䏍系。近 年来, 亚洲和大洋洲各国家及地 区在这一领域取得了长足进步。 儿理神经免疫学研究协会于 2012 年 成立了官方的中国地区分会, 促 进了该领域的科学发展, 并逐渐 扩展到整个亚洲和大洋洲地区。 扩展到整个亚洲和大洋洲地区。 本文记录了其成长历程, 以及中
国、中国台湾地区、澳大利亚和 日本最近取得的研究进展。

\section{RESEARCH EXPENDITURE}

生物医学研发的支出反映了国家 的富裕水平及对公民健康的重视 程度。除国家/地区的总支出外, 支出的增长趋势也是衡量发展的 重要指标。最新汇总的2007到 2012这6年的数据说明, 欧洲各国 家及地区和美国的公共和私人科 研支出均有下降, 但曰本、中国 台湾地区、印度、澳大利亚、新 加坡、韩国和中国却有增加。由 手对生物医学的投次加大, 亚太 地区的心理神经免疫学研究蓬勃 发展。

亚洲和大洋洲各国家及地区生物 医学研究的日益繁荣增强了各国 之间对一流科研人才吸引的竞争 力, 这为在海外深造的亚洲和大 洋洲的科学家回到自己祖国工作 提供了更多的机会。他们中的许 多人与曾经一起深造的西方科学 家制定了合作研究计划, 为亚洲 和大洋洲以外国家的科学家创造 了更多赴海外工作的机会。因 此, 亚洲加大对生物医学研究的 投资为世界各地的科学家增加了 机会, 推进了生物医学领域的新 发现
PNIRS China

中国和西方国家的生物医学研究 工作相对独立, 直到21世纪初才有 改变。儿理神经免疫学研究协会 (PNIRS) 是1993年成立的全球性非营 利组织, 致力于推进对神经系统与 免疫系练之间相互作用以及对行为 与健康之间父系的研究。由于中国 生物医学椟究的薄勃发展, PNA 生物医学研究的蓬勃发展, PNIS 于2012年成立了中国分会

中医(TCM)强调身心平衡, 即脑 激素和免疫系统之间的信息交 流。中医已成为现代心理神经免 疫学理论的基础, 因此, 东西方 科学家之间的合作使人们能够更 好地理解人类的使康问题。2013 好地理解人类的健康回题。2013

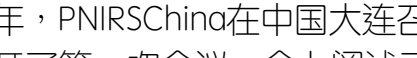
开了第一次会议, 会上阐述了 PNIRSChina的三个主要目标: (1) 进行可转化为临床应用的基础研 究; (2) 促进不同学科的科学家之 间的联系; (3) 建立心理神经免疫 学研究者的学术交流平台。协会成 立了一个委员会来组织研讨会和 排其他推广剒施, 供中西方科学安

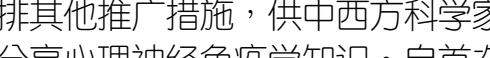
分字心理神经免投学知识。目首次 会议以来, 协会在中国各地又举行 了六场研讨会

\section{《脑、行为和免疫》}

《脑、行为和免疫》(Brain, Behavior, and Immunity)于2000年 成为PNIRS的官方杂志。多年来, 直在全球免度学和神经科学期 直在全球免投学和神经科学期

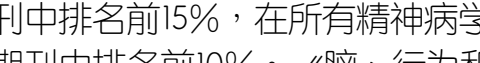
期刊中排名前 $10 \%$ 。《脑、行为和 免疫》一直被全球公认为“神经科学 中最好的免疫学杂志”。最初, 美 国科学家是该杂志的主要撰稿人。 现在, $17 \%$ 的投稿来自中国, 成为 该杂志投稿量第二多的国家。通过

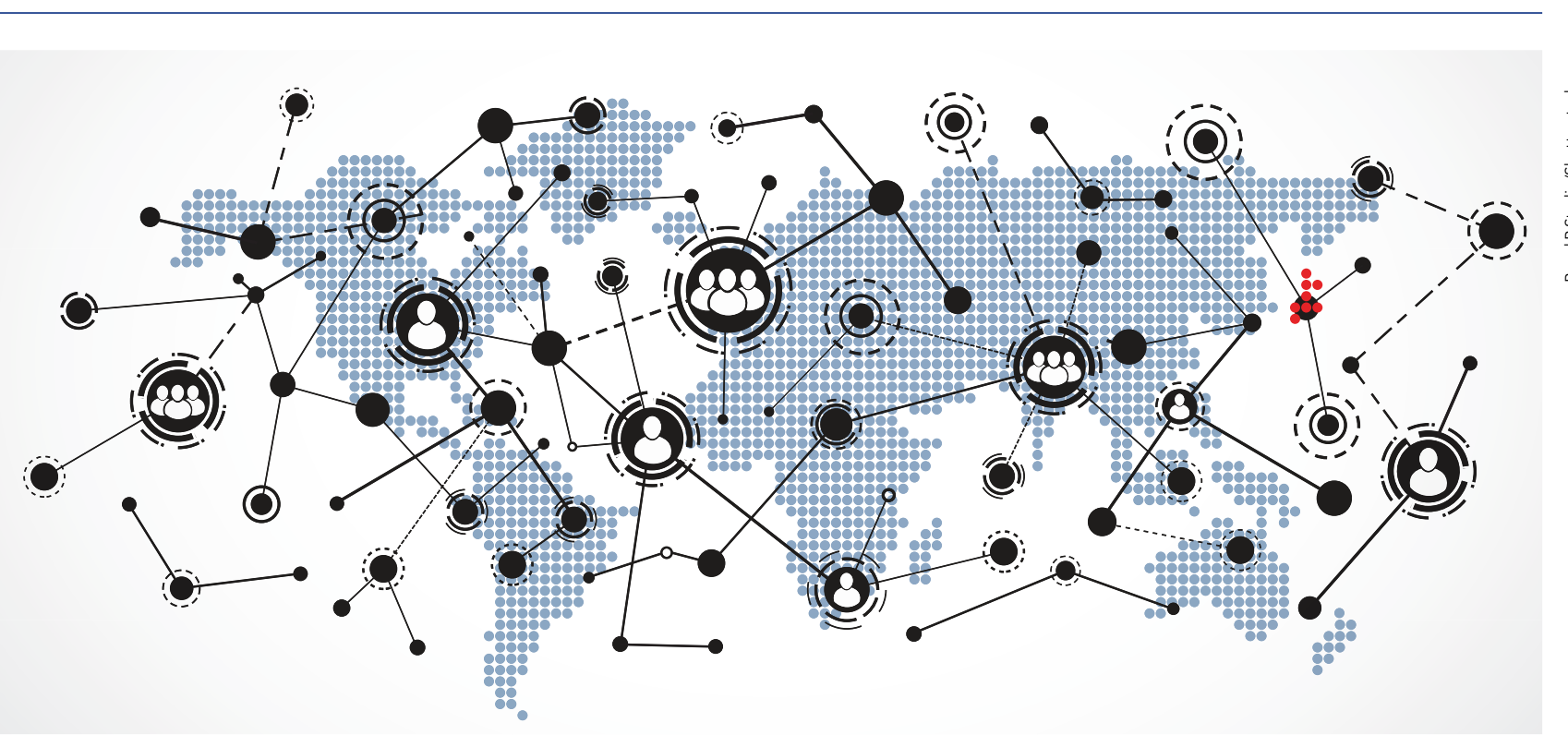

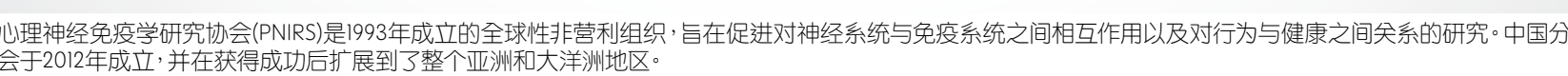

引用频次可以说明, 中国学者发表 的论文质量很高（2015年至2016年 间引用量最高的10篇2014年论文中 就有3篇来自中国实验室)

PNIRS $_{\text {Asia-Pacific }}$

在中国成功举办了七场研讨会之 后, 协会扩展到整个亚洲和大洋 洲, 包括北亚、南亚、东南亚国 家和地区以及澳大利亚和新西兰。 因此, PNIRS China 于 2017 年更名为 PNIRSAsia-Pacific。此后, 协会在 中国大陆以外的国家和地区（包括 中国台湾地区、澳大利亚、日本、 韩国和新西兰）又举办了九场研讨 会。所有研讨会的摘要以及演讲者 的照片可在线查看, 网址为: www.pnirs.org/pnirsasia-pacific

\section{全球研究网}

根据博士后培训、合作研究和科学 会议的数据显示, 尽管心理神经免 疫学的研究正在 全球范围内展 开, 但很少西 方科学家化大量 时间在亚洲工 作 $\circ$ PNIRSAsia-
Pacific委员会率 先提出了一项 计划, 将亚洲

之为 PNIRS $_{\text {Asia-Pacific }}$ Global Research Connections (全球研究网) 。协 会开发了一个网站, 感兴趣的科学 家只需提供一些简单的信息即可加 入该计划。该计划的目标是将亚太 各国家及地区的心理神经免疫学科 学家联系在一起, 并与世界各地的 实验室合作, 以促进全球生物医学 研究。任何感兴趣的科学家都可以 在这里注册: https://pnirs.ansc. illinois.edu/\#

中国

由于医学研发的支出增加, 中国在 顶级科学期刊和生命科学出版物上 发表论文的数量现在分别排名第 和第三。中国科学院目前是全球声 望排名第五的科研机构。屠织叭幼教 授于2015年成为中国第一位犾得诺 贝尔生理学或医学奖的学者。她对 中草药具有浓厚的兴趣, 经大量计 究发现了一种被称为青高素的植物 PNIRS $_{\mathrm{A}}$ 委员会开创 项计划, 将亚洲和大洋洲的心理。医学汼重将精神和 神经免疫学实验室与其他国家的 离的解剖结构。此 实验室联系起来。类疗法旨在恢复整 与其他国家的实验室联系起来, 称疾的微生物。中医是世界上最古老了有力支持。但与大型随机对照试
的医学之一, 讲究身心平衡。中医 想和推拿等。中西医结合将替代疗 法与传统医学相结合, 涵盖生活方

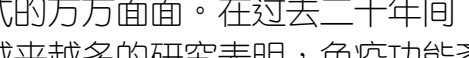
乱可能是神经系统疾病的表现, 而 神经系统常地会昌致免度功能障 碍。这方面的一些重要研究源自中 国, 并发表在《脑、行为和免疫》 杂志上。

\section{中国台湾地区}

在过去的33年中, 台湾科学家在 䈐论文行免没》上发表了33

两年间发表的, 说明是在过去的 免疫学研究员上升趋势。科学家纽

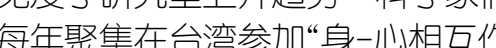
用国除的相互作 分享神经科学的 研究成果。本协会鼓励通过临床疾 病研究来探寻全球化的医疗方案。 全球化的医疗方案。 了法包括太极拳、草药、针灭、冥 


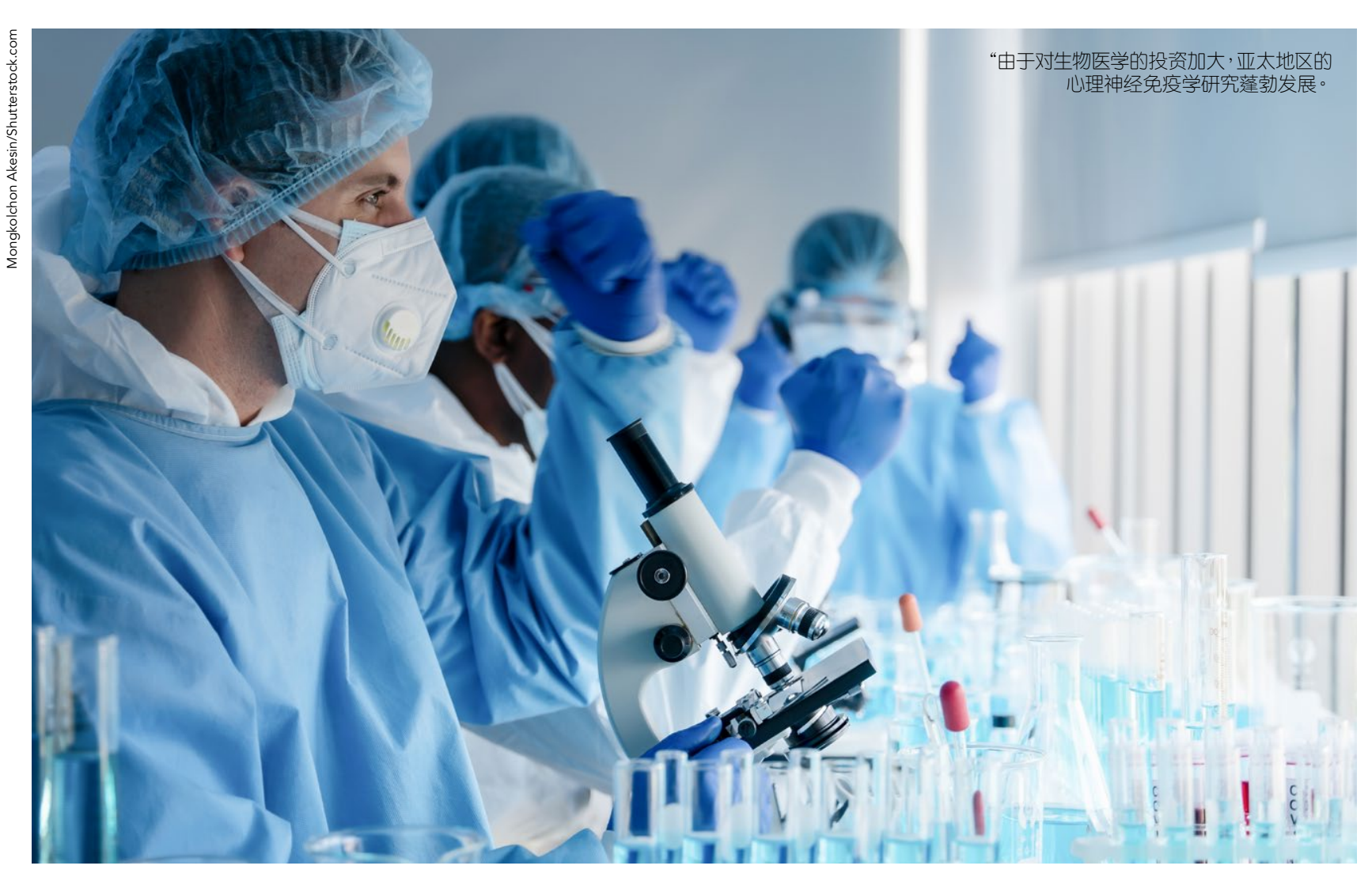

面对冠状病毒疫情, 我们比以往任 何时候都更加需要促进全球合作, 以发现造福全球健康的解决方案。

验相比, 单一生活方式干预效果的 检测难度更大。因此，东西方生物 医学合作可借助先进的科学方法,

通过临床试验为传统医学疗法提供 支持。

\section{澳大利亚}

澳大利亚仍是推进PNIRSAsia-

Pacific目标的主要参与者。自1987 年心理神经免㡯学诞生以来, 澳大 利亚一直从事该领域的研究。澳大 利亚在《脑、行为和免疫》杂志上 发表的论文数排多第四, 论文

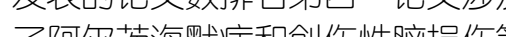

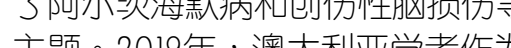
主题。2019 年 澳大秒学者作为 第一作者或资深作者在《脑、行为 和免疫》上发表了16篇论文・另有 篇论文是澳大利亚人作为合作者 发表的, 至少有10个国际合作研究 才引参与其中。重要的是, 其中多 篇论文堪称当前心理神经免疫学研
究全球合作的典范。若干篇论文由 五个以上国家或地区的作者共同发 表, 整合了研究技术、研究理念 学者和学生的研究经验, 取得了仅 靠一个研究小组无法实现的研究成 果。《脑、行为和免疫》杂志的两 位副主编是澳大利亚科学家, 多次 研讨会也展示了澳大利亚的心理神 经免疫学研究成果。

日本

日本神经免疫学会成立于1988年, 由专门研究神经疾病的医师组成。 该学会和PNIRSAsia-Pacific计划在 未来举办联合研讨会, 重点探讨 相䏍研究。Tadamitsu Kishimoto 等日本免疫学家在分子免疫学领域 获得了突破性的成果。他的工作促 使研发了一种重要的抗体（一种 能够攻击病毒、细菌和其他化学物 质的蛋白质），可用于治疗类风
湿䏌节炎 (免疫系统侵蚀矣节的 疾病)。令人矣注的是, 由于这 种抗体可抑制免疫系统的某些功 能, 因此该抗体也已被批准作为 SARS-COV-2的治疗药进入临床试 验, SARS-COV-2是引起当前冠状 病毒大流行的病毒

心理神经免疫学应更加专业化 以造福全球人类健康。在研究肠免疫一脑轴中取得的激动人心的新 解古代 社 会支持网络对丝激和儿理健康管 理意义重大。空气污染在全球范 围内影䛛着心理神经免疫, 但具 的全球化, 我们在理解生活环境 了进步。鉴于当前的冠症症取 需要促进全球合作, 以找到解决 疫学感兴趣的科学家架起沟通的 桥梁, 引领全球心理神经免疫学 研究 体污染类型可能因国家及地区而 异。得益于儿理神经免疫学研究 如何影响脑和身体的机制方面取得 了进鉒于当前的冠状病毒疫 情 我们侯都更力 全球健康问题的方案。PNIRSAsiaPacific继续为东西方对心理神经免

\section{研究背后}

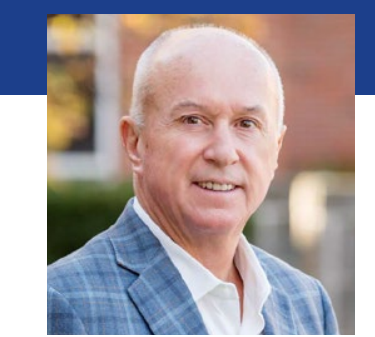

Keith W.

Kelley

电子邮件:

@illinois.edu

电话:

网址:

www.orcid.org/0000-

\subsection{3 \\ 研究目标}

Kelley博土率先为心理神经免疫学研究协会提出促进东西方合作的理念。

\section{详细介绍}

Bio

Keith W. Kelley是伊利诺伊大学名誉教授。他连续 30 年获得美国国立卫生研究院 $(\mathrm{NIH})$ 的资助, 发表了 350 多篇同行评审的科学论文和专著章节。Kelley教 授于2003年至2017年担任 《大脑 “行为和免疫》的 总编辑。《大脑、行为和免疫》在所有免疫学、神经 科学和精神病学期刊中排名前 $15 \%$. Kelley博士为 PNIRS 提出了促进东西方交流的理念, 并继续领导 组织整个亚洲的 PNIRS Asia-Pacific 协作研讨会。

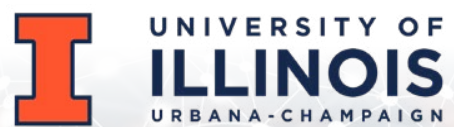

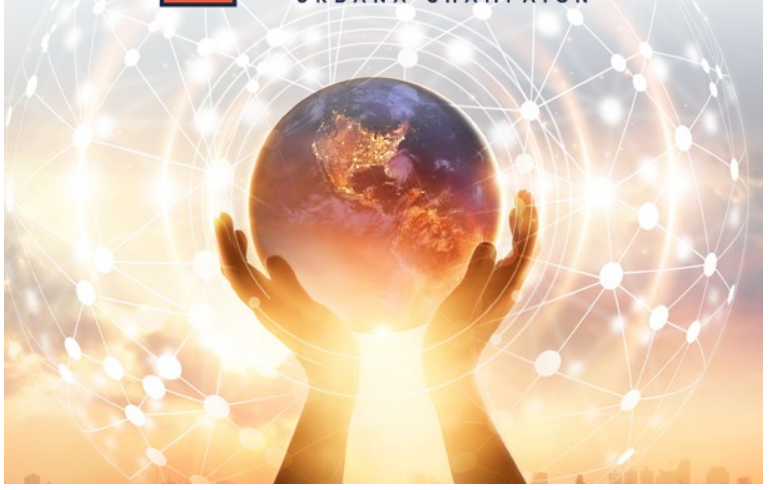

参考 进和推广

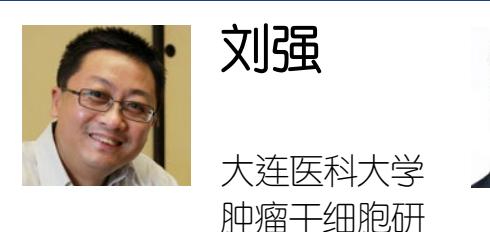
旅顺南路9号癌症研究中心 317 室, 邮编116001。

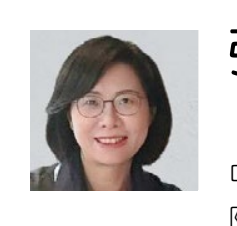

张㦟芝

中国医药大学 附设医院身心 介面研究中心, 台湾台中。 究院, 中华人民共和国大连
2 Mark R. Hutchinson 阿德莱德大学阿 德莱德医学院, 澳大利亚南澳大利亚州阿德 莱德市5006 , 以及纳米级生 物光子学卓越研究中心。

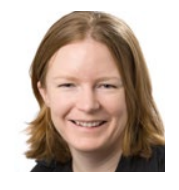

Sarah J. Spencer 皇家墨尔本理工 大学健康与生物

医学院
大利亚维多利亚州
Kelley, K.等人(2020)心理神经免疫学走向东方: PNIRS China 分会的发展及其扩展为 PNIRS $_{\text {Asia-Pocific }}$ 《大脑、行为和免疫》 https://doi.org/10.1016/j. bbi.2020.04.027

\section{个人回复}

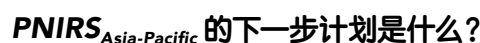

U 尽管新冠疫情对PNIRSAsia-Pacific的目标构成, 重重挑战, 但我们仍在制定前进的计划。我们将 继续寻找机会来发掘那些希望进一步了解免疫系 统与大脑相互作用领域最新发现的新成立科学协 会。PNIRSAsia-Pacific委员会还将确定专家发言人 并为其年度会议组织一次研讨会。我们将会使用

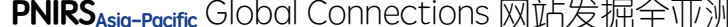
的生物医学研究科学家, 从而建立一个广污的数腒 库。亚洲和全世界科学家之间的合作研穷将得到促

Keith W. Kelley 和共同作者. U 